\title{
FEATURES OF HEMOSTASIS IN DIFFERENT TYPES OF 24 HOURS DAY'S PROFILE OF BLOOD PRESSURE IN HYPERTENSIVE PATIENTS OF DIFFERENT AGE GROUPS
}

\author{
V. S. Pasko
}

State institution of science «Research and practical center of preventive and clinical medicine» State administrative department, Kyiv , Ukraine

\begin{abstract}
Summary
Given the fact that not only in the absence of control over hypertension and failure to achieve target blood pressure levels, but also with different daily blood pressure profiles, there is a high probability of thrombotic complications. That is why it was important for us to find out the peculiarities of the state of platelet aggregation indicators depending on 24 hours day's profile of blood pressure. To date there are only isolated reports of hemostasis in this category of individuals. We conducted such studies in middle-aged and elderly patients.

The aim of the study was to identify the baseline levels of hemostasis indices depending on 24 hours day's profile of blood pressure in middle-aged and elderly hypertensive patients.

Material and methods. We performed the comparative assessment of status of hemostasis in 30 middle-aged (group I) and 30 elderly patients (group II) respectively to clarify this issue in our work. Patients of the surveyed groups were comparable in age, sex and related pathology. Control group consisted of 15 patients for every of the surveyed groups (group III the middle-aged and group IV the elderly respectively) matched with basic by age and gender.

Conclusions. Thus, in hypertensive patients there is an increase in platelet activity with age which is more pronounced when stimulating ADP in non-dipper group compared to dipper. In addition in older patients the most significant changes are determined in spontaneous and adrenaline-induced aggregation compared with middle-aged patients.

In most patients of both groups revealed changes in hemostasis in the direction of increasing the aggregation properties of platelets. However, more pronounced trends in such changes were found in patients with insufficient blood pressure at night.
\end{abstract}

Keywords: arterial hypertension, essential hypertension, hemostasis.

The objective of the study. To identify the baseline levels of hemostasis indices depending on 24 hours day's profile of blood pressure (BP) in middle-aged and elderly hypertensive patients.

Material and methods. We performed the comparative assessment of status of hemostasis in 30 middle-aged (group I) and 30 elderly hypertensive patients (group II) respectively to clarify this issue in our work. Patients of the surveyed groups were comparable in age, sex and related pathology. The control group consisted of 15 patients for every of the surveyed groups (group III the middle-aged and group IV the elderly respectively) matched with basic by age and gender. Control was presented with patients without arterial hypertension who were hospitalized with diagnoses of chronic gastritis, duodenitis, cholecystitis, atherosclerotic cardiosclerosis, with angina functional class that is not above I, without cardiac arrhythmias and heart failure that is not above II A stage for Strazhesko M. D.- Vasylenko V. Kh.

The diagnosis and stage of essential hypertension was set according to the criteria of WHO and the International Society of Hypertension (2013). Patients with significant heart rhythm disorders, angina functional class that is above I, heart failure that is above II A stage for Strazhesko M. D.- Vasylenko V. Kh. were not involved in the study. Cases with symptomatic hypertension and obesity more than the second degree were also excluded.

Spontaneous and induced platelet aggregations were determined. Platelets were activated with adenosine di- 
phosphate (ADP) $\left(1 \times 10^{-5} \mathrm{mM} / \mathrm{L}\right)$, adrenaline $(1 \mu \mathrm{g} / \mathrm{mL})$ and analysed by optical aggregometry (SOLAR AP-2110). We determined and analyzed the following indices: amplitude of aggregation (AA) (\%), time of maximal aggregation (TMA) (min.), slope of aggregation (SA) (\%/min.). Plasma haemostasis was defined with hemocoagulometer Amelung KC 1A.

Results and discussion. The results of induced platelet aggregation in middle-aged hypertensive patients showed a significant increase in aggregation activity in both dipper and non-dipper groups due to AA of patients by $91,7 \%$ and $132 \%$ respectively. In dipper group AA determined by ADP was 1,9 times and in non-dipper group 2,3 times higher than the normative indicators. In two groups a significant acceleration of the aggregation process was determined by the indicators of SA by $105,5 \%$ and $112 \%$ respectively. Thus, in dipper group ADP-induced AA was $53,3 \pm 20,3 \%$; in non-dipper group there was a more significant increase in this indicator to $64,5 \pm 33,5 \%$ against $27,8 \pm 1,9 \%$ of control $(\mathrm{p}<0,05)$ which was $21 \%$ more.

\section{The induced platelet aggregation indices depending on 24 hours day's profile of blood pressure in middle-aged hypertensive patients and control $(\mathrm{M} \pm \sigma)$}

\begin{tabular}{|c|c|c|c|c|c|}
\hline \multirow{2}{*}{\multicolumn{2}{|c|}{$\begin{array}{l}\text { The indices } \\
\text { The inductors }\end{array}$}} & \multicolumn{3}{|c|}{ The distribution of the surveyed groups } & \multirow{2}{*}{$\mathbf{p}$} \\
\hline & & Dipper & Non-dipper & Control & \\
\hline \multirow{2}{*}{$\mathrm{AA}(\%)$} & ADP & $53,3 \pm 20,3$ & $64,5 \pm 33,5$ & $27,8 \pm 1,9$ & $\begin{array}{l}\mathrm{p}_{1}<0,05 \\
\mathrm{p}_{2}<0,05 \\
\mathrm{p}_{3}<0,001\end{array}$ \\
\hline & Adrenaline & $31,1 \pm 16,5$ & $41,1 \pm 14,7$ & $35,2 \pm 2,6$ & $\begin{array}{l}\mathrm{p}_{1}>0,05 \\
\mathrm{p}_{2}>0,05 \\
\mathrm{p}_{3}>0,05\end{array}$ \\
\hline \multirow{2}{*}{ TMA (min.) } & $\mathrm{ADP}$ & $3,3 \pm 1,7$ & $3,9 \pm 1,6$ & $4,7 \pm 1,0$ & $\begin{array}{l}\mathrm{p}_{1}>0,05 \\
\mathrm{p}_{2}>0,05 \\
\mathrm{p}_{3}>0,05\end{array}$ \\
\hline & Adrenaline & $4,2 \pm 2,3$ & $5,8 \pm 3,4$ & $4,9 \pm 0,2$ & $\begin{array}{l}\mathrm{p}_{1}>0,05 \\
\mathrm{p}_{2}>0,05 \\
\mathrm{p}_{3}>0,05\end{array}$ \\
\hline \multirow{2}{*}{$\mathrm{SA}(\% / \mathrm{min})}$. & $\mathrm{ADP}$ & $56,3 \pm 14,8$ & $58,1 \pm 30,8$ & $27,4 \pm 10,7$ & $\begin{array}{l}\mathrm{p}_{1}<0,05 \\
\mathrm{p}_{2}<0,05 \\
\mathrm{p}_{3}<0,01\end{array}$ \\
\hline & Adrenaline & $16,1 \pm 6,9$ & - & $15,4 \pm 1,3$ & $\begin{array}{l}\mathrm{p}_{1}>0,05 \\
\mathrm{p}_{2}>0,05 \\
\mathrm{p}_{3}>0,05\end{array}$ \\
\hline
\end{tabular}

Remark: $\mathrm{p}_{1}$-statistical significance of difference between dipper and non-dipper, $\mathrm{p}_{2}$-statistical significance of difference between dipper and control, $\mathrm{p}_{3}$-statistical significance of difference between non-dipper and control.

We found unidirectional changes in platelet functional activity in middle-aged hypertensive patients: the increase in AA with ADP-induced aggregation in non-dipper group by $21 \%(\mathrm{p}<0,05)$ compared with dipper group and the acceleration of SA by $3,2 \%(p<0,05)$.

Indicators of spontaneous aggregation of elderly hypertensive patients were also determined depending on 24 hours day's profile of BP.

When comparing the values of platelet hemostasis (Table 2) in patients of both groups there is a significantly higher level (1,7 times) of the rate of spontaneous aggregation in individuals of non-dipper group compared with dipper group $(4,2 \pm 1,6 \%$ against $2,5 \pm 0,2 \%$; $<<0,05)$.
In patients of non-dipper group aggregation indices showed a moderate increase in AA by $68 \%$.

Studying the indicators of induced platelet aggregation in elderly hypertensive patients depending on 24 hours day's profile of BP (Table 3 ) we found the significant increase in ADP-induced platelet aggregation in patients of non-dipper group $(74,7 \pm 25,1 \%$ against $51,4 \pm 22,2 \%$; $\mathrm{p}<0,05)$ and epinephrine-induced TMA $(8,2 \pm 2,6$ minutes vs $5,7 \pm 2,5$ minutes; $p<0,05)$.

Also with adrenaline-induced aggregation there was the significant reduction in TMA by $30,5 \%(p<0,05)$ in dipper group. 
The indices of the spontaneous platelet aggregation depending on 24 hours day's profile of blood pressure in elderly hypertensive patients and control $(\mathrm{M} \pm \sigma)$

\begin{tabular}{|c|c|c|c|c|}
\hline \multirow{2}{*}{ The indices } & \multicolumn{2}{|c|}{ The distribution of the surveyed groups } & \multirow{2}{*}{$\mathbf{p}$} \\
\cline { 2 - 5 } & $\begin{array}{c}\text { Dipper } \\
\text { (n=15) }\end{array}$ & $\begin{array}{c}\text { Non-dipper } \\
\text { (n=15) }\end{array}$ & $\begin{array}{c}\text { Control } \\
\text { (n=15) }\end{array}$ & \multicolumn{1}{c|}{$\begin{array}{c}\mathrm{p}_{1}<0,05 \\
\mathrm{p}_{2}>0,05 \\
\mathrm{p}_{3}>0,05\end{array}$} \\
\hline \multirow{2}{*}{ AA (\%) } & $2,5 \pm 0,7$ & $4,2 \pm 1,6$ & $2,5 \pm 0,2$ & $\begin{array}{l}\mathrm{p}_{1}>0,05 \\
\mathrm{p}_{2}>0,05 \\
\mathrm{p}_{3}>0,05\end{array}$ \\
\hline TMA (min.) & $6,7 \pm 3,4$ & $6,9 \pm 3,7$ & $8,8 \pm 0,8$ & $\begin{array}{l}\mathrm{p}_{1}>0,05 \\
\mathrm{p}_{2}>0,05 \\
\mathrm{p}_{3}>0,05\end{array}$ \\
\hline
\end{tabular}

Remark: $\mathrm{p}_{1}$-statistical significance of difference between dipper and non-dipper, $\mathrm{p}_{2}$-statistical significance of difference between dipper and control, $\mathrm{p}_{3}$-statistical significance of difference between non-dipper and control.

\section{The induced platelet aggregation indices depending on $\mathbf{2 4}$ hours day's profile of blood pressure in elderly hypertensive patients and control $(\mathrm{M} \pm \sigma)$}

\begin{tabular}{|c|c|c|c|c|c|}
\hline \multirow{2}{*}{\multicolumn{2}{|c|}{$\begin{array}{l}\text { The indices } \\
\text { The inductors }\end{array}$}} & \multicolumn{3}{|c|}{ The distribution of the surveyed group } & \multirow[b]{2}{*}{$\mathbf{p}$} \\
\hline & & $\begin{array}{c}\text { Dipper } \\
(n=15)\end{array}$ & $\begin{array}{c}\text { Non-dipper } \\
(\mathrm{n}=15)\end{array}$ & $\begin{array}{c}\text { Control } \\
(\mathrm{n}=15)\end{array}$ & \\
\hline \multirow{2}{*}{$\mathrm{AA}(\%)$} & ADP & $51,4 \pm 22,2$ & $74,7 \pm 25,1$ & $35,0 \pm 4,9$ & $\begin{array}{c}\mathrm{p}_{1}<0,05 \\
\mathrm{p}_{2}<0,001 \\
\mathrm{p}_{3}<0,01\end{array}$ \\
\hline & Adrenaline & $49,1 \pm 16,9$ & $56,5 \pm 52,4$ & $42,9 \pm 5,6$ & $\begin{array}{l}\mathrm{p}_{1}>0,05 \\
\mathrm{p}_{2}>0,05 \\
\mathrm{p}_{3}>0,05\end{array}$ \\
\hline \multirow{2}{*}{ TMA (min.) } & ADP & $4,9 \pm 3,1$ & $5,4 \pm 2,8$ & $6,9 \pm 1,4$ & $\begin{array}{l}\mathrm{p}_{1}>0,05 \\
\mathrm{p}_{2}>0,05 \\
\mathrm{p}_{3}>0,05\end{array}$ \\
\hline & Adrenaline & $5,7 \pm 2,5$ & $8,2 \pm 2,6$ & $8,2 \pm 1,8$ & $\begin{array}{l}\mathrm{p}_{1}<0,05 \\
\mathrm{p}_{2}<0,05 \\
\mathrm{p}_{3}<0,05\end{array}$ \\
\hline \multirow{2}{*}{$\mathrm{SA}(\% / \mathrm{min})}$. & ADP & $65,7 \pm 26,2$ & $62,2 \pm 20,3$ & $49,9 \pm 11,8$ & $\begin{array}{l}\mathrm{p}_{1}>0,05 \\
\mathrm{p}_{2}>0,05 \\
\mathrm{p}_{3}>0,05\end{array}$ \\
\hline & Adrenaline & $20,1 \pm 10,0$ & $23,4 \pm 19,9$ & $18,0 \pm 1,5$ & $\begin{array}{l}\mathrm{p}_{1}>0,05 \\
\mathrm{p}_{2}>0,05 \\
\mathrm{p}_{3}>0,05\end{array}$ \\
\hline
\end{tabular}

Remark: $\mathrm{p}_{1}$-statistical significance of difference between dipper and non-dipper, $\mathrm{p}_{2}$-statistical significance of difference between dipper and control, $\mathrm{p}_{3}$-statistical significance of difference between non-dipper and control.

In most patients of both groups revealed changes in platelet hemostasis in the direction of increasing the aggregation properties of platelets. However, more pronounced trends in such changes were found in patients with insufficient BP at night. This was manifested by an increase in platelet AA determined with ADP by $45,3 \%(\mathrm{p}<0,05)$ in non-dipper group compared with dipper group. TMA with adrenaline stimulation in non-dipper group increased by $43,9 \% ; \mathrm{p}<0,05$ which indicated the activation of platelet hemostasis in these patients at night.
It was important to determine the manifestations of plasma hemostasis depending on 24 hours day's profile of BP. The data are presented in table 4.

Analysis of plasma hemostasis in middle-aged hypertensive patients in dipper group showed a significant slowing by $36,1 \%(\mathrm{p}<0,05)$ of fibrinolysis activity compared with control group. Thus, fibrinolysis was $215,0 \pm 49,7$ minutes while in control group $158,0 \pm 5,7$ minutes. 
The indicators of plasma hemostasis depending on 24 hours day's profile of blood pressure in middle-aged hypertensive patients and control $(\mathrm{M} \pm \sigma)$

\begin{tabular}{|c|c|c|c|c|}
\hline \multirow[b]{2}{*}{ The indices } & \multicolumn{3}{|c|}{ The distribution of the surveyed group } & \multirow[b]{2}{*}{$\mathrm{p}$} \\
\hline & $\begin{array}{l}\text { Dipper } \\
(n=15)\end{array}$ & $\begin{array}{l}\text { Non-dipper } \\
(\mathrm{n}=15)\end{array}$ & $\begin{array}{c}\text { Control } \\
(n=15)\end{array}$ & \\
\hline $\mathrm{FG}(\mathrm{g} / \mathrm{L})$ & $3,1 \pm 0,8$ & $2,8 \pm 0,9$ & $3,1 \pm 0,2$ & $\begin{array}{l}\mathrm{p}_{\mathrm{p}}>0,05 \\
\mathrm{p}_{2}>0,05 \\
\mathrm{p}_{3}>0,05\end{array}$ \\
\hline Fibrinolysis (min.) & $215,0 \pm 49,7$ & $174,2 \pm 13,9$ & $158,0 \pm 5,7$ & $\begin{array}{l}\mathrm{p}_{1}>0,05 \\
\mathrm{p}_{2}<0,05 \\
\mathrm{p}_{3}>0,05\end{array}$ \\
\hline AT-III (\%) & $80,7 \pm 7,3$ & $79,6 \pm 9,1$ & $93,8 \pm 6,9$ & $\begin{array}{l}\mathrm{p}_{1}>0,05 \\
\mathrm{p}_{2}>0,05 \\
\mathrm{p}_{3}>0,05\end{array}$ \\
\hline
\end{tabular}

Remark: $\mathrm{p}_{1}$-statistical significance of difference between dipper and non-dipper, $\mathrm{p}_{2}$-statistical significance of difference between dipper and control, $\mathrm{p}_{3}$-statistical significance of difference between non-dipper and control.

Thus, in middle-aged patients of non-dipper group an imbalance in the coagulation and fibrinolytic activity of blood was detected. This is consistent with the literature on the peculiarities of the hemostasiological picture in hypertensive patients with taking into account 24 hours day's profile of BP in the direction of hypercoagulation $[1,2,3,4,5,6,7]$.

So, in different profiles of BP erasure of age differences on the majority of indicators of spontaneous aggregation of thrombocytes is noted.

Conclusions. In hypertensive patients there is an increase in platelet activity with age which is more pro- nounced when stimulating ADP in non-dipper group compared to dipper. In addition in older patients the most significant changes are determined in spontaneous and adrenaline-induced aggregation compared with middle-aged patients.

In most patients of both groups revealed changes in hemostasis in the direction of increasing the aggregation properties of platelets. However, more pronounced trends in such changes were found in patients with insufficient blood pressure at night.

Conflict of interest. The authors declare no conflicts of interest.

\section{ЛITEPATУPA}

1. Баркаган 3.С., Момот А. П. Диагногстика и контролируемая терапия нарушений гемостаза. Москва: Ньюдиамед. 2001. 296 с.

2. Братчик А. М. Клинические проблемы фибринолиза. К.: Здоровья. 1993. 343 с.

3. Современные представления о системе гемостаза / Волков Г. Л. И др. Киев: Наукова думка. 2005. 296 c.
4. Грицюк А.И., Амосова Е. Н., Грицюк И. А. Практическая гемостазиология. К.: Здоров'я. 1994. 256 с.

5. Иванов Е. П. Диагностика нарушений гемостаза. Минск: Беларусь. 1983. 223с.

6. Коркушко О.В., Коваленко А. Н. Система свертывания крови при старении. К.: Здоровье. 1988. 216 с.

7. Hypertension and hemostatic/fibrinolytic balance disorders / Tabak O. at el. Clin. Invest. Med. 2009. № 32 (6). P. 285-292.

\section{REFERENCES}

1. Barkagan, Z.S., Momot, A.P. (2001). Diagnogstika i kontroliruyemaya terapiya narusheniy gemostaza [Diagnostics and controlled therapy of hemostasis disorders]. Moskva: N'yudiamed, 296.
2. Bratchik, A.M. (1993). Klinicheskiye problemy fibrinoliza [Clinical problems of fibrinolysis]. K.: Zdorov'ya, 343.

3. Volkov, G.L., Platonova, T.N., Savchuk, A.N., Gornitskaya, O.V., Chernyshenko, T.M., Krasnobrizhaya, 
Ye.N. (2005). Sovremennyye predstavleniya o sisteme gemostaza [Modern ideas about hemostasis system]. Kiev: Naukova dumka, 296.

4. Gritsyuk, A.I., Amosova, Ye.N., Gritsyuk, I.A. (1994). Prakticheskaya gemostaziologiya [Practical hemostasiology]. K.: Zdorov’ya, 256.

5. Ivanov, Ye.P. (1983). Diagnostika narusheniy gemostaza [Diagnostics of hemostatic disorders]. Minsk: Belarus', 223.
6. Korkushko, O.V., Kovalenko, A.N. (1988). Sistema svertyvaniya krovi pri starenii [Blood coagulation system during aging]. K.: Zdorov'ye, 216.

7. Tabak, O., Gelisgen, R., Uzun, H., Kalender, B., Balci, H., Curgunlu, A., Simsek, G., Karter, Y. (2009). Hypertension and hemostatic/fibrinolytic balance disorders. Clin. Invest. Med., 32 (6), 285-292.

\section{Резюме}

\section{ОСОБЛИВОСТІ ГЕМОСТАЗУ ПРИ РІЗНИХ ПРОФІЛЯХ АРТЕРІАЛЬНОГО ТИСКУ У ХВОРИХ НА ГІПЕРТОНІЧНУ ХВОРОБУ РІЗНИХ ВІКОВИХ ГРУП \\ В. С. Пасько}

Державна наукова установа «Науково-практичний центр профілактичної та клінічної медицини» Державного управління справами, м. Київ, Україна

При відсутності контролю над артеріальною гіпертензією і недосягненні цільових рівнів артеріального тиску, а також при різних добових профілях, існує висока ймовірність тромботичних ускладнень. Тому нам важливо було з'ясувати особливості стану показників агрегації тромбоцитів залежно від добового ритму артеріального тиску. На сьогодні існують лише поодинокі повідомлення щодо особливостей тромбоцитарного гемостазу у хворих середнього і похилого віку.

Мета - виявлення вихідного рівня показників тромбоцитарного гемостазу при різних профілях артеріального тиску у хворих середнього і похилого віку на гіпертонічну хворобу II стадії.

Матеріал і методи. У нашій роботі ми проводили порівняльну оцінку стану гемостазу у 30 хворих середнього (група I) та 30 похилого віку (група II) відповідно. Контрольну групу склали по 15 хворих для кожної з обстежених категорій (відповідно III група - обстежені середнього віку та IV група - обстежені похилого віку) з порівнянним статевим складом.

Висновки. У хворих на гіпертонічну хворобу II стадії з віком відбувається збільшення тромбоцитарної активності, що найбільш виразно проявляеться при стимуляції ААФ у групі non-dipper порівняно 3 dipper. Крім того, у хворих старшої вікової групи найбілыш суттєві зміни визначаються при спонтанній та алреналін-індукованій агрегації у порівнянні з хворими середнього віку.

У більшості хворих обох груп виявлені зміни тромбоцитарного гемостазу у бік підвищення агрегаційних властивостей тромбоцитів. Однак, більш виразні тенденції таких змін були виявлені у хворих із недостатнім зниженням артеріального тиску в нічний час.

Ключові слова: артеріальна гіпертензія, гіпертонічна хвороба, гемостаз. 


\section{Резюме}

\section{ОСОБЕННОСТИ ГЕМОСТАЗА ПРИ РАЗНЫХ ПРОФИЛЯХ АРТЕРИАЛЬНОГО ДАВЛЕНИЯ У БОЛЬНЫХ ГИПЕРТОНИЧЕСКОЙ БОЛЕЗНЬЮ РАЗНЫХ ВОЗРАСТНЫХ ГРУПП

\author{
В. С. Пасько
}

Государственное научное учреждение «Научно- практический центр профилактической и клинической медицины» Государственного управления делами, г. Киев, Украина

Не только при отсутствии контроля над артериальной гипертензией и недостижении целевых уровней артериального давления, а и при разных суточных профилях существует высокая вероятность тромботических осложнений. Вот почему нам важно выяснить особенности состояния показателей агрегации тромбоцитов в зависимости от суточного ритма артериального давления. На сегодня существуют лишь единичные сообщения об особенностях тромбоцитарного гемостаза у этой категории лиц. Такие исследования нами были проведены у больных среднего и пожилого возраста.

Цель - выявление исходного уровня показателей гемостаза при разных профилях артериального давления у больных среднего и пожилого возраста гипертонической болезнью II стадии.

Материал и методы. Аля выяснения этого вопроса в нашей работе мы проводили сравнительную оценку состояния гемостаза у 30 больных среднего (группа I) и 30 пожилых дюдей (группа II) соответственно. Контрольную группу составили по 15 больных для каждой из обследованных категорий (соответственно III группа - обследованные среднего возраста и IV группа - обследованные пожилого возраста) с сопоставимым половым составом.

Выводы. Таким образом, у больных гипертонической болезнью II стадии происходит увеличение тромбоцитарной активности с возрастом, наиболее отчетливо проявляется при стимуляции ААФ в группе non-dipper по сравнению с dipper. Кроме того у больных старшей возрастной группы наиболее существенные изменения определяются при спонтанной и алреналин-индуцированной агрегации по сравнению с больными среднего возраста.

У большинства больных обеих групп выявлены изменения тромбоцитарного гемостаза в сторону повышения агрегации тромбоцитов. ОАнако, более выразительные тенденции таких изменений были обнаружены у больных с недостаточным снижением артериального давления в ночное время.

Ключевые слова: артериальная гипертензия, гипертоническая болезнь, гемостаз.

Інформація про авторів знаходиться на сайті http://www.cp-medical.com.

Дата надходження до редакції - 22 травня 2021 року 